\title{
A Useful and Safe Method for Retrieving a Round Metallic Object from an Airway
}

Daisuke Jingu ${ }^{1}$, Akira Horii ${ }^{1}$, Yu Matsuzono ${ }^{1}$, Satoshi Ubukata ${ }^{1}$, Kosuke Sato $^{1}$, Makoto

Shoji ${ }^{1}$, Noriko Kondo ${ }^{1}$, Hiroshi Takahashi ${ }^{1}$, and Hiroshi Watanabe ${ }^{1}$

${ }^{1}$ Affiliation not available

September 11, 2020

\begin{abstract}
We experienced a case with accidental aspiration of a metallic round foreign body. As there is no established techniquefor retrieving a metallic round ball from an airway, we propose that endoscopic net forceps with the support of a laryngeal mask airway is the best choice for such occasions.
\end{abstract}

\section{Key Clinical Message}

The endoscopic net forceps with the support of a laryngeal mask airway is a dependable choice for retrieving a round metallic object from an airway

\section{KEYWORDS}

airway foreign body, laryngeal mask, metallic round body, net forceps

\section{CLINICAL IMAGE}

An 85-year-old man with a 2-day history of dyspnea and general malaise was admitted to our hospital with hypopnea and acidosis. Chest radiograph and CT scan identified a round high-density shadow about $1 \mathrm{~cm}$ in diameter (Figure 1). We deduced that a pachinko-ball (Pachinko is a Japanese pinball gambling game) obstructed the right inferior lobar bronchus. Forced coughing and back blow failed to remove it. To remove the obstacle with minimal invasion, we considered several devices with an endoscope under mechanical ventilation; a magnet, a basket type or a pentatope type grasping forceps, or an endoscopic net forceps, with the support of a laryngeal mask airway, which we selected. The pachinko ball was successfully retrieved without severe damage to the patient (Figure 2). The patient received non-invasive positivepressure ventilation for one day and antibiotic administration for 14 days. Rehabilitation improved his general condition, and he was transferred to a long-term hospital on day 48. There is no consensus on how to retrieve a metallic round ball from an airway. Based on our experience, we propose that endoscopic net forceps with the support of a laryngeal mask airway is a dependable choice for such occasions.

199 words

\section{CONSENT STATEMENT}

Informed consent was obtained from the patient for this study.

\section{ACKNOWLEDGMENTS}

We are grateful to Barbara Lee Smith Pierce (Retired Professor, University of Maryland University College) for her editorial work in the preparation of the manuscript. 


\section{CONFLICT OF INTEREST}

None declared.

\section{AUTHOR CONTRIBUTIONS}

DJ, YM, SU, KS, MS, HT, HW, and NK substantially contributed to the diagnosis and clinical care of the patient. DJ wrote the draft of the manuscript, and DJ and AH critically revised it for important intellectual content. HW gave final approval of the version to be published.

\section{FUNDING INFORMATION}

None.

\section{ORCID}

Daisuke Jingu https://orcid.org/0000-0002-0153-3912

Akira Horiihttps://orcid.org/0000-0002-3967-3291

\section{REFFERENCES}

No references.

\section{Legend to Figures}

\section{Figure 1}

Chest radiograph (A) and CT scan (B) show a round, high density shadow nearly $1 \mathrm{~cm}$ in diameter. The right inferior lobar bronchus seems to be obstructed by a metallic round substance.

\section{Figure 2}

(A) Bronchoscopic examination revealed complete obstruction of the right inferior lobar bronchus by a metallic round foreign body.(B) The foreign body was successfully removed by endoscopic net forceps with the support of a laryngeal mask airway. The foreign body was a pachinko-ball.

\section{Hosted file}

figure v1.0_20200822_revision.pptx available at https://authorea.com/users/358108/articles/ 480405-a-useful-and-safe-method-for-retrieving-a-round-metallic-object-from-an-airway 